\title{
COGNITION IN PEOPLE WITH PRADER-WILLI SYNDROME: INSIGHTS INTO GENETIC INFLUENCES ON COGNITIVE AND SOCIAL DEVELOPMENT
}

\section{Joyce Whittington* and Anthony Holland}

\author{
University of Cambridge, Department of Psychiatry, \\ Cambridge, UK
}

\footnotetext{
*Dr JE Whittington

University of Cambridge

Department of Psychiatry

Douglas House

18b Trumpington Road

Cambridge CB2 8AH

Tel: 01223746108

Fax: 01223746122

e-mail: jew1000@cam.ac.uk
} 


\begin{abstract}
We present a mini-review of cognition in Prader-Willi syndrome. Studies cited include findings on general ability (IQ), IQ correlates with family members, strengths and weaknesses in cognitive profiles in genetic subtypes, attainment in literacy and numeracy, language, comprehension, modality preferences, executive functions, and social cognition. The latter includes investigations of theory of mind, emotion recognition, face processing and knowledge of social norms. Results from research on mouse models and brain imaging studies relevant to cognition are briefly discussed.

The importance of these studies to understanding and managing education and behaviour in PWS and the limitations of the studies in terms of small numbers, non-representativeness, and lack of replication is also touched upon.
\end{abstract}

Key words Prader-Willi syndrome, cognition, ability, attainment, social cognition 


\section{Introduction}

The dictionary defines cognition as 'knowledge in its widest sense'. As such it encompasses, not only intellectual abilities and attainments, but also social abilities and understanding. Human cognition has been studied largely for three main reasons: for scientific understanding, for insights into learning and education, and to inform our understanding of social behaviour. In the typically developing population there is an extensive literature on all such aspects of cognition and how cognitive profiles vary but additional unique insights into all aspects of cognition can be gained by examining how cognition is altered in groups whose development is atypical, such as those with specific genetic syndromes. While educational and social implications will be similar, in the sense of what particular educational or social profiles follow from given cognitive abilities, strengths or weaknesses, there will be an added dimension to scientific enquiry knowing the genetic origin (genotype) of that syndrome. The new questions then for psychology and neuroscience are 'How does the known genetic abnormality affect developmental brain processes and how do these, in turn, result in systematic changes in cognition that are characteristic of that particular group’? The first steps to progress is a characterization of the cognitive findings. We describe what progress has been made in the scientific understanding of the atypical cognition, and its educational and social consequences, in the genetically determined neurodevelopmental disorder, Prader-Willi Syndrome (PWS). The references cited include preferentially those where PWS has been determined by genetic testing and not by clinical features only. Many early reports are unreliable because the diagnoses of PWS of those included in the studies were largely by clinical criteria only. In addition, because of the small sample sizes in many studies there is a need for replication of the findings. The focus of this article is inevitably on the cognitive deficits associated with PWS but it is also important to acknowledge that individuals with PWS have strengths as well as weaknesses - these are also commented on. We first give a broad overview of PWS and then consider the different components of the cognitive phenotype, including social cognition We go on to look at how the manipulation of key 'PWS genes' affect aspects of cognition in mouse models of the syndrome.

Finally we review the evidence for brain abnormalities associated with cognition in PWS.

\section{Overview of PWS}

PWS has a birth incidence rate of 1:20,000 to 1:25,000, and a population 
prevalence of about 1:50,000. The syndrome results from the loss of expression of paternally expressed genes from the PWS imprinted cluster in the q11-13 region of the paternally inherited chromosome 15 . The loss is due to a deletion of part, or all, of this region (deletion subtype) or to the inheritance of two maternally marked chromosome $15 \mathrm{~s}$ and no paternally marked copy (uniparental disomy - UPD subtype). Coded in this 'critical region' of chromosome 15 are both imprinted and non-imprinted genes (see Fig 1), SNORD 116, Magel 2 and IPW being the genes whose absence of expression at the locus 15q11-13, singularly or in combination, are considered central to PWS. Whilst the different genetic sub-types described have a common genotype, that is the absence of expression of the paternally related alleles of maternally imprinted genes at 15q11-13, there are genetic differences between the sub-types that might account for the phenotypic differences that are observed between the different genotypes (see Hoybye chapter 2 (2014) for full details of the genetics of PWS). Imprinted genes are known to be actively expressed in the foetus and the placenta (Keverne, 2015) and in PWS this is apparent in the fact that there is a recognizable phenotype at birth (see below). Whilst many of the major criteria of the phenotype described above, and some additional aspects (e.g. poor temperature control, sleep abnormalities), can be attributed to abnormalities in hypothalamic development and functioning, the reason for the cognitive impairments remains uncertain.

\section{FIGURE 1}

As with other genetically determined neurodevelopmental syndromes, PWS is associated with a particular pattern of cognitive and social development and an increased risk for specific co-morbid behavioural and psychiatric problems (Hoybye 2013 chapters 6-7). What is referred to as the 'behavioural phenotype' of PWS emerges with development. There is an initial extreme hypotonia and failure to thrive followed in early childhood by hyperphagia, developmental delay, evidence of relative growth and sex hormone deficiency (short stature and impaired sexual development); and a marked propensity to problem behaviours, such as temper outburst, repetitive and ritualistic behaviours and skin picking (Whittington and Holland 2004). Whilst those with the genetic sub-types of PWS have the core features of PWS in common, there are differences depending on whether the person has PWS due to a deletion or a UPD, and within the deletion subgroup whether the deletion is larger (Type 1, between breakpoints 1 and 3) or smaller (Type 2, between breakpoints 2 and 3).

\section{Cognition in PWS}


Cognitive impairments in people with PWS include low IQ for family background and, in particular, difficulties with abstract ideas and comprehension. In addition, social cognition is usually impaired and peer group relationships may be poor or absent, presenting in a similar manner to some of the features associated with autism spectrum conditions. As discussed in detail below, compared to the general population, performance on tasks of executive function are also affected with specific deficits, for example, in task switching that may also be associated with repetitive symptoms, such as repetitive questioning, and aversion to changes in routine. Caution is needed in looking at specific individuals with PWS. Whilst, in many studies, the cognitive impairments and associated behavioural problems have been reported to be characteristic of people with PWS, they vary in severity from person to person. As in the general population, such differences may reflect differences in familial genetic background and/or environment. These observations from systematic research studies are important indicators of common consequences of PWS but their salience needs to be determined for each individual and not assumed. These different aspects are now considered in greater detail.

\section{General Ability (IQ)}

Several studies, not primarily concerned with general ability, have reported FSIQ scores in small samples of people with PWS. Here we describe studies whose primary aims were either to investigate cognitive ability in PWS or to investigate differences between genetic subtypes including ability differences. We found only one study (Dykens et al. 1992) that looked at the stability of IQ, and which showed stability over periods ranging from 1 year to over 9 years, (mean $3.25+2.30$ ) but not all participants were genetically confirmed to have PWS. The 31 study participants included 15 tested twice with the age-appropriate Wechsler scales and 16 tested twice with the Stanford-Binet scale (see Table 1).

TABLE 1

TABLE 2

Table 2 documents other studies of IQ in people with PWS. The first was a population study with genetic confirmation of PWS and comprised all ages from 4 years. The distribution of IQ was found to be roughly normal. The second was an early study of children in which the diagnosis was based on clinical criteria. The third was designed to investigate genetic subtype differences, especially deletion subtypes, on a range of intellectual and behavioural measures. The other two 
were based on clinic populations and reported median and interquartile ranges. Four studies reported profiles of subtest scores as well as FSIQ, VIQ and PIQ. The three Western studies found VIQ higher in UPD and PIQ higher in deletion subtypes, but both were higher in the Japanese deletion group. This may have reflected sampling or ethnic differences. In the UK population-based study the profiles of average scores across the battery of tests were quite different for the two sub-types, with statistically significant differences for the Vocabulary (UPD higher) and Coding (deletion higher, also found in the Copet et al. 2010 study) tests.

The Coding test measures processing speed and a similar difference was found between genetic sub-types on a physiological measure of speed, namely, the time to maximum evoked potential response to a stimulus (Stauder et al. 2005). In the population sample, those with deletions also had higher average scores than those with UPD on the visuo-spatial tests Block Design and Object Assembly, suggesting a difference between those with the different genetic subtypes in visuo-spatial ability. A further difference was found between people with the two main genetic sub-types when the correlations between PWS and sibling IQs were investigated (Whittington et al. 2009). The usual correlation (Paul 1980) of 0.5 between sibling pairs was found between those with PWS due to UPD ( $n=10)$ and their siblings but the correlation between those with PWS due to a deletion $(n=18)$ and their siblings was zero. The correlation between all non-PWS sibling pairs in this study was the expected 0.5. Unlike the UK study of IQ, which was representative of a population, this research was a self-selected sample and needs replication.

In a study of IQ in related individuals, Malich et al. (2000) reported the FSIQ scores of 22 people with PWS, assessed by the Swiss version of the Wechsler scales or the Kaufman Assessment Battery for Children, and their mothers, assessed by the short form of the Wechsler test (WASI). There were 18 with the deletion subtype and 4 with UPD. The correlation between all PWS and mothers' IQ was $0.33,0.58$ for males and 0.12 for females; for a control group of typically developing children the correlations with mothers' IQ were 0.46 overall, 0.63 for males and 0.29 for females.

The similarities and differences between the genetic sub-types outlined in this section indicate that loss of maternally imprinted genes on chromosome 15 results in lowered IQ scores in all people with PWS, presumably due to structural alterations in the developing brain, but that genetic differences between the subtypes, such as enhanced expression of paternally imprinted genes (in those with UPD), modifies or adds to these structural alterations, possibly by strengthening or weakening links between brain areas. Interestingly, in the brain, it is paternally imprinted genes (ie. those genes where the allele of maternal origin 
is the only allele expressed) that are preferentially expressed in the cortex (Keverne et al. 1996).

\section{Attainment and specific abilities}

Attainment in basic literacy and numeracy in PWS is very variable, as reported by Whittington et al. (2004b). As expected, in the UK population study, for 45 people with PWS aged greater than 7 years, attainment was correlated with general ability (FSIQ), with correlations of $0.60,0.66$ and 0.53 for reading, spelling and arithmetic. Attainment declined with age, correlations with age were $-0.32,-0.31$ and -0.42 for reading, spelling and arithmetic. These correlations were in the opposite direction to that of IQ with age ( $r=0.21 \mathrm{~ns}$ ), illustrating the maxim 'Use it or lose it'. This study found underachievement particularly among people who had attended special schools for children with moderate or severe disabilities. The authors speculated that this could be due to low expectations of teachers and an emphasis on practical skills aimed at the majority of children who had physical disabilities. Studies of attainment in maths, reading, spelling and language are presented in Table 3.

\section{TABLE 3}

Attainment in maths, reading, spelling and language

Most researchers report numerical skills to be one of the most problematical areas of cognitive functioning for people with PWS. This was the case in the population-based study of Whittington et al (2004). and in the studies by Bertella et al. (2005) and Semenza et al. (2008). In the UK population study 16 participants could not do any calculation. Semenza et al (2008). were particularly interested in genetic subtype differences but their results seemed to show more similarities, with parity judgements, division and approximations very poor in both groups. The largest differences they found was worse multiplication-related items in the UPD group. Butler et al. (2004) looked at differences between three genetic subtypes: type1 deletions (T1), type2 deletions (T2), and UPD. On the Woodcock Johnson Psycho-Educational Battery, the UPD and T2 groups were much better than the $\mathrm{T} 1$ group on the math cluster and applied problems, while T2>UPD>T1 on calculations. Since the T1 and T2 groups did not differ significantly on the IQ measures reported, it seems that the loss of additional imprinted genes in the T1 group gives rise to lower achievement, either directly or perhaps indirectly via more behavior problems. 
In the above study, Butler et al.(2004) also looked at reading scores. They found that the T2 and UPD groups again scored much higher than the T1 group on the reading cluster, letter-word identification and reading comprehension. Thus lower achievement in this group extends to reading as well as mathmatics. In all groups, reading comprehension was lower than other attainment scores, reflecting the poor comprehension seen in PWS (see below). In the Whittington et al.(2004) study thirteen PWS participants could not read at all. Only one report of spelling achievement was identified, the UK population study, which found scores slightly below those for reading, and again thirteen people could not spell at all. Language development is usually delayed and often complicated by difficulties in articulation (possibly related to hypotonia), usually requiring speech therapy (Whittington et al. 2002). Both the Chen et al.(2010) and Dimitropoulos et al. (2013) studies found expressive language was poorer than receptive language.

\section{Comprehension}

Many parents report that their child has difficulty in understanding what is said to them and often 'gets hold of the wrong end of the stick'. This is partly due to the tendency to interpret everything literally and partly to the tendency to 'concrete' thinking and processing. Failure to progress to a more abstract phase of development could be due to their relatively low general ability. A lack of understanding may also underlie a particular behaviour observed in people with PWS. When performing a variety of tasks they were observed to discard partially correct solutions and start the task again from scratch. These tasks included: addition of two numbers (in which both numbers were counted on the fingers, rather than 'counting on'); the Wechsler Object Assembly and Block design tests (where a correct partial assembly was scrapped when the next piece tried seemed not to fit); the DKEFS (Delis-Kaplan Executive Function System) Word Context task (where the previous clues were ignored and new guesses were made to the current clue alone). One young man executed addition of two, two-digit numbers perfectly but when confronted by addition of two three-digit numbers said, 'I can’t do those - nobody showed me how' (all personal observations). Personal observation has also shown that people with PWS have difficulties with perseveration when generating examples or reasons (e.g. verbal fluency tests; the Comprehension test of the Wechsler intelligence scale).

\section{Modality differences}

A paper by Stauder et al. (2002) reported greater impairment in the auditory modality in PWS in an event-related brain activity study. The participants were 10 adults with PWS,ages 30.8+7.1 years, and 10 adult normal controls, ages 23.2 \pm 6.9 years, who participated in visual (on a computer monitor) and auditory (via 
headphones) oddball tasks in which they had to press a button to the appearance of the rarer of three regular stimuli, with a 'novel' stimulus presented once in each trial. EEG recordings were obtained from a cap of 30 electrodes. Although the PWS group showed strongly reduced P3 activity in both modalities, it was much worse in the auditory modality.

Savantism - skill with jigsaw puzzles

The clinical diagnostic criteria for PWS include 'exceptional skill with jigsaw puzzles'. Investigations of this claim (Verdine et al. 2008) have led to the findings that people with PWS seem to enjoy jigsaws and spend a lot of time doing them (hence part of the claim is due to practice), that people with PWS use the shape of the pieces rather than the picture or colour as their main strategy (so they out-perform other people only when using blank shapes), and that this advantage disappears when pieces are cut by straight lines rather than the traditional jigsaw shapes. This reported exceptional skill is more common in those with delPWS.

\section{Executive Functioning (EF)}

Measures of EF in PWS have reported low scores relative to the general population. Table 4 lists investigations of executive functions in people with PWS.

\section{TABLE 4}

As the table shows, a wide variety of tests purporting to measure various executive functions were used, with comparison groups ranging from the general population norms (Jauregi 2007), typically developing individuals (Chevalere 2015 and Woodcock 2009), people with Fragile X syndrome (Woodcock 2009), groups matched on verbal ability (Walley 2005), to no comparison group (Whittington, unpublished). In the Walley and Donaldson study, when genetic sub-types (12 deletion, 6 mUPD) were considered separately, there was a trend for the deletion sub-type to perform better on the visuospatial planning task, namely the Tower of London. This study suggests that future assessments of executive function in PWS should distinguish between genetic sub-types. Comparison of the Verbal Fluency letter scores with those of the Whittington study showed a discrepancy in the scores of their deletion groups for the letters $\mathrm{F}$ and A. Jauregi et al. 2007 reported that, in assessments of memory, sequential processing appeared to be more severely affected than simultaneous processing. Simple reaction time, choice reaction time, trail making test B, copying and recall 
of the Rey-Osterrieth complex figure were significantly correlated with PIQ; phonetic fluency and semantic fluency were significantly correlated with VIQ. In the Woodcock et al. study, both PWS and FraX groups were impaired relative to controls but when IQ and age were factored out, the PWS group was still impaired on task switching. Chevalere et al. 2015 reported similar findings of impairment relative to controls and, again, after controlling for FSIQ, impairment remained for switching and also for cognitive estimation. In the DKEFS (DelisKaplan Executive Function System) word context test, the meaning of a nonsense word is to be deduced from a series of increasingly explicit clues. After each clue, the participant is asked to guess the meaning of the nonsense word. In an unpublished study (JEW), it was observed that many participants ignored previous clues and guessed only on the basis of the current one, even when reminded of the previous ones.

\section{Social Cognition}

Some of the social difficulties in PWS are similar to those seen in people with autistic spectrum conditions. In a recent review (Bennett et al 2015) over 25\% of people with PWS met criteria for ASD, with a greater percentage in the UPD subtype. However, whereas impaired social cognition is highlighted in autism, social difficulties in PWS are not well researched, especially the role of social cognition in these difficulties. People with PWS appear to have abnormalities in their perception of other people and in understanding the behaviour and mental states of others, as shown in studies investigating theory of mind (ToM), emotion recognition, face processing and knowledge of social norms.

\section{TABLE 5}

Table 5 summarises studies of social cognition in people with PWS. Three studies looked at ToM in PWS; all provide evidence that ToM is poorly developed. The third study suggested that scores improve with age and with receptive vocabulary. In the study of causal attribution, the PWS group showed a greater understanding of desire than emotion or cognitive motivations. The third and fourth studies in the table show that matching emotions depicted in facial photographs and naming such emotions result in quite different orders of difficulty for the various emotions; it seems that fear is easiest to match but happiness is easiest to name. In the fourth study there were no genetic subtype differences in scores overall or for individual emotions, and correlations with age did not reach significance, although there was a tendency for scores to decrease with age. The Halit et al.(2008) study of face recognition showed a deficit on this task with 7 of 16 
scores outside the normal range. Two studies used the N170 (face sensitive) component in electrophysiological (ERP) investigations of face processing. In PWS, faces elicited an N170 that was similar in time course and scalp distribution to that of typical adults. Both studies found differences between the genetic subtypes in amplitude and latency to upright and inverted faces. Koenig et al. (2004) investigated social attribution in three groups of participants: PWS, pervasive developmental disorder (PDD) and an age-matched control group. Overall the PWS group performed similarly to the PDD group and significantly worse than the IQ-matched group Some studies mention impaired observance of social norms in people with PWS. Dimitropoulos et al. 2013 reported responses of parents/carers of people with PWS to questions about their social behavior, some of which are presented in Table 4. Invading others' personal space, difficulties with turn taking and interrupting convesations were also mentioned in the study by Lo et al. (2013) cited previously.

In unpublished work, our group used the first four Dewey stories (Dewey 1991) to investigate knowledge of social norms among 37 people with PWS due to a deletion, ages 6-51, and 14 UPD PWS, ages 11-50. These short stories describe the reasoning and actions of young men in social situations. After each action in the story participants are asked to rate the action as normal, slightly odd, very odd or shocking. It was clear from the responses that people with PWS have little idea of social norms. For example, when a girl tells a young man who has been following her closely round a supermarket to 'buzz off', 16 (43\%) with the deletion and five (39\%) UPD said the behavior was shocking; when a young man picks up an unknown baby from a pram and checks its clothing, 13 (35\%) people with PWS deletions and 3 (21\%) with UPD said the behavior was normal. Perhaps unsurprisingly for PWS, 10 people (eight (22\%) deletion , two (14\% UPD) said that feeding bits of lunch to pigeons in a park was shocking.

\section{Mouse models and cognition}

Research on mouse models of PWS began in 1992, and most early models looked at physical and behavioural characteristics, particularly feeding behavior, size and weight, and hypotonia. Cognitive characteristics, such as spatial learning and memory, were later added to mouse model research. Because of the loss of expression of multiple imprinted genes in PWS, mouse models with knock out of single imprinted genes have also been used to try to tease out the contribution of each of these genes to the total PWS phenotype. The single gene models that have addressed cognitive issues are described below. 
Relkovic et al. (2012) investigated the influence of palatable food reward on learning in an imprinting centre deletion mouse model of PWS (PWS-IC). They found that on a nonspatial maze-based task the PWS-IC mice reached criteria quicker with fewer errors during acquisition and also reversal learning. Devaluing the reinforcer impaired wild type performance but had no effect on PWS-IC mice. This is reminiscent of the cognitive effort put into food acquisition by some people with PWS.

In an earlier paper, Relkovic et al. (2010) used a five-choice serial reaction time task (5-CSRTT) to show that PWS-IC mice have reduced attentional capabilities. Although these mice reached criteria levels (>30 completed trials, $>80 \%$ accuracy, $<25 \%$ omissions), they showed decreased accuracy, increased omissions and longer correct reaction times compared with wild type mice. Under reduced stimulus duration, considered to tax attentional functioning, they showed increased deficits relative to controls. However, premature responses, motoric function (trial number, nose pokes, panel pushes) and motivation (latency to collect and consume reward) were not significantly different between groups. These observations are similar to those of people with PWS.

Meziane et al. (2015) reported a deficit in social recognition and social interaction and a reduced learning ability in adult male mice lacking the Magel2 gene (M2). Daily administration of oxytocin in the first postnatal week was reported to prevent deficits in social behavior and learning abilities in $\mathrm{M}^{-}$mice. The social tasks were time spent with a conspecific grid or conspecific_animal (less time for M2); the recognition memory task was relative time spent exploring familiar and new objects (more time on familiar for $\mathrm{M}^{-}$); spatial learning was measured by the Morris water maze test (higher latencies and distances to the hidden platform, reduced preference for the target quadrant and reduced number of platform position crosses for the $\mathrm{M}^{-}$mice). However, working memory measured in the Y-maze was not affected. There were no cognitive impairments in female $\mathrm{M}^{-}$ mice. Very similar social deficits have been reported by Dombret et al. (2012) for a Maged1 knock-out mouse model. The authors speculated that the oxytocin deficiency is responsible for the social deficits seen in autism and PWS. Muscatelli et al. (2000) assessed spatial learning and memory in Necdin deficient mice using the Morris water maze in which animals have to locate a hidden platform using spatial cues. After 9 days of training, Necdin deficient mice showed decreased escape time and decreased path length relative to wild type mice, although performances were no different at the commencement of training. In the habituation phase, before training, the Necdin deficient mice eventually spent more time on or in the vicinity of the platform. The authors deduce that the mutants had better memories for the platform location. They speculate that the 
performance of the mutant mice is similar to the strengths in visual processing tasks and long term memory seen in PWS.

Ding et al. (2008) investigated memory and learning in mice with a deletion of the $40+$ copies of the SnoRNA, Snord116. They reported that they found no differences in rates of spontaneous alternation in the Y maze between the deletion and wild type mice at age three months, indicating normal short term working memory. In the novel arm preference test at age five months, there was no difference from wild type mice in the numbers of entries into the novel arm. The authors concluded that the deletion mice have no obvious defect in working memory or spatial memory. During six days of training on the accelerating rotarod, the wild type mice improved significantly whereas the learning curves of the deletion mice remained essentially flat, indicating a motor learning deficiency. Together these mouse modela are beginning to suggest which of the imprinted genes in PWS may contribute to the cognitive strengths and weaknesses observed in people with PWS.

\section{Brain imaging and cognition}

Brain imaging studies of people with Prader-Willi syndrome are still in their infancy; most have concentrated on structural brain differences between PWS and typical development. These have recently been reviewed by Manning et al (2015). Several have looked at brain areas involved in eating behavior; few have mentioned any aspect of cognition. There are two main drawbacks to brain imaging studies: the speculative nature of links between any brain abnormalities found and specific PWS characteristics, and the small sample sizes used. Table 6 presents brain imaging studies in which authors have speculated that their results may be relevant to cognition. The table illustrates the diversity of investigations used, the small numbers of participants involved, and the lack of replication.

\section{TABLE 6}

These different research findings demonstrate the beginnings of an understanding of the cognitive deficits in PWS. The low IQ for family background may be related to lower brain volumes and lower cortical complexity in certain brain areas, specific deficits in executive functions and language relative strengths and weaknesses may be localized to specific brain areas, as may cognitive differences between the genetic subtypes. However, because of the small sample sizes, independent replication of these results is needed before they can be fully accepted. 


\section{Conclusions}

It is clear from the literature that the absence of expression of specific maternally imprinted gene(s) located at 15q11-13 have a significant impact on intellectual and social cognition in both obvious and also in more subtle ways. However, this picture is more complicated for two main reasons. First, the nature and extent of such impairments vary within the groups that have been investigated with, for example, some people with PWS having FSIQ scores well into the mild intellectual disability range and others having scores that are within what is considered the expected range for the general population. Secondly, there is an additional and not properly understood influence of the different genetic sub-types of PWS on these profiles. As a general rule those with UPD would appear to be more atypical, particularly because of their slower processing speed. However, from the perspective of the FSIQ scores of those with UPD, correlations with other first degree family members was found to be 0.5 , which is what is to be expected. This may not be the case for those with delPWS where the correlation with siblings' FSIQ was found to be 0 .

From the perspective of supporting people with PWS to lead more independent lives, these observations are very important for several reasons. First, detailed assessment of these various domains of functioning can help characterise and make sense of some of the everyday functional difficulties that a person with PWS may experience. Sound and informed cognitive assessment identifying impairments in such tasks as attention and the ability to re-focus on another task, or on planning ability or social functioning, enable family members and those supporting people with PWS to have some understanding of what it is like for that person trying to function in a cognitively demanding and frequently changing world. Secondly, such cognitive difficulties may help to explain behavioural problems and ultimately it maybe by seeking to intervene at the cognitive level that behavioural problems can be ameliorated. Thirdly, such observations challenge us to develop day-to-day strategies that help to compensate for such difficulties. For example, the use of visual timetables and other visually orientated communication strategies may improve understanding, reduce arousal, and minimise the likelihood of temper outbursts, particurly in the deletion subtype. Finally, the evidence that functional abilities may decline with age (as suggested by a cross-sectional study) does suggest that people with PWS should be living lives where there are opportunities to maintain functional and educational skills. We need to be much more sophisticated in drawing upon such research both to inform educational strategies during childhood and the nature and structure of support environments in adult life. 
Research on cognition in PWS is hampered by lack of replication, small sample sizes due to the rarity of the condition, and hence non-representative samples. However, there do seem to be some tentative conclusions to be drawn. With regard to education, average IQ is in the low 60s, so children are likely to need extra help and a slower pace to learn; deletion subtypes seem to prefer the visual mode; disomy subtypes have slower processing speeds. With regard to social behaviours, poor recognition of emotion and poor understanding of social behavioural norms lead to impaired peer relationships. Difficulties with executive functions, such as task switching, may be associated with temper outbursts and adherence to routines. With regard to progress on the main scientific question, it seems that the genetic abnormality leads to both structural and connectivity changes in the developing brain (see Manning et al 2015 for review) which just might be related to particular alterations in cognition (language areas and sustained attention, respectively). 


\section{Funding}

The research did not receive any specific grant from funding agencies in the public, commercial, or not-for-profit sectors. 


\section{REFERENCES}

Bennet JA, Germani T, Haqq AM, Zwaigenbaum L (2015) Autism spectrum disorder in Prader-Willi syndrome: a systematic review. Am J Med Genet A 167A:2936-44

Bertella L, Girelli L, Grugni G, Marchi S, Molinari E, Semanza C (2005) Mathematical skills in Prader-Willi syndrome $J$ Intellect Disabil Res 49:159-169

Butler MG, Bittel DC, Kibiryeva N, Talebizadeh Z, Thompson T (2004) Pediatrics 113:565-74

Chen CM, Chen CL, Hou JW, Hsu HC, Chung CY, Chou SW, Lin CH, Chen KH. (2010) Developmental profiles and mentality in preschool children with Prader-Willi syndrome: a preliminary study. Chang Gung Med. J. 33:436-42.

Chevalere J, Postal V, Jauregui J, Copet P, Laurier V, Thuilleaux D (2015) Executive functions and Prader-Willi syndrome: global deficit linked with intellectual level and syndrome-specific associations Am J on Intellect \& Dev Disabil 120:215-229

Copet P, Jauregi J, Laurier V, Ehlinger V, Arnaud C, Cobo AM, Molinas C, Tauber M, Thuilleaux D. (2010). Cognitive profile in a large French cohort of adults with Prader-Willi syndrome: differences between genotypes. J. Intellect. Disabil. Res. 54:204-15.

Curfs LM, Wiegers AM, Sommers JR, Borghgraef M, Fryns JP. (1991) Strengths and weaknesses in the cognitive profile of youngsters with Prader-Willi syndrome. Clin. Genet. 40:430-4.

Dewey M (1991) Living with Asperger syndrome. In: Frith U (ed) Autism and Asperger syndrome. Cambridge CUP

Dimitropoulos A, Ferranti A, Lemler M (2013) Expressive and receptive language in Prader-Willi syndrome: report on genetic subtype differences. J Communication Disorders 46:193-201

Dimitropoulos A, Ho A, Feldman B (2013) Social responsiveness and competence in Prader-Willi syndrome:direct comparison to Autism Spectrum Disorder J Autism Dev Disord 43:103-113

Ding F, Li HH, Zhang S, Solomon NM, Camper SA, Cohen P Franke U (2008) SnoRNA Snord116 (Pwcr1/MBll-85) deletion causes growth deficiency and hyperphagia in mice PLOS ONE 3:e1709

Dombret C, Nguyen T, Schakman O, Michaud JL, Hardin-Pouzet H, Bertrand JM, De Backer O (2012) Loss of Maged1 results in obesity, deficits of social interactions, impaired sexual behavior and severe alteration of mature oxytocin production in the hypothalamus. Hum Mol Genet 21:4703-17

Dykens EM, Hodapp RM, Walsh K, Nash LJ (1992) Profiles, correlates, and trajectories of intelligence in Prader-Willi syndrome. J Am Acad child Adolesc Psychiatry 31:1125-29 
Ekman P. \& Friesen W.V. (1976) Pictures of Facial Affect.Consulting Psychologists Press, Palo Alto, CA.

Halit H, Grice SJ, Bolton P Johnson MH (2008) Face and gaze processing in PraderWilli syndrome $J$ of Neuropsychology 2:65-77

Hashimoto T, Mori K, Yoneda Y, Yamaue T, Miyazaki M, Harada M, Miyoshi H, Kuroda Y (1998) Proton magnetic resonance spectroscopy of the brain in patients with Prader-Willi syndrome Pediat Neurol 18: 30-35

Honea R, Holsen LM, Lepping RJ, Perea R, Butler MG, BrooksWM, Savage CR (2012) The neuroanatomy of genetic subtype differences in Prader-Willi syndrome Am J Med Genet B 159B:243-253

Hoybye C (ed) (2013) Prader-Willi Syndrome. Nova Science Publishers, New York

Jauregi J, Arias C, Vegas O, Alen F, Martinez S, Copet P, Thuilleaux D. (2007) A neuropsychological assessment of frontal cognitive functions in Prader-Willi syndrome. Journal of Intellectual Disability Research 51:350-365.

Keverne E. B., Fundele R., Narasimha M., Barton S. C.\& Surani M.A. (1996) Genomic imprinting and the differential roles of parental genomes in brain development. Brain Research: Developmental Brain Research 92, 91-100

Keverne E B (2015) Genomic imprinting, action, and interaction of maternal and fetal genomes Proc Natl Acad Sci U S A. 2015 ;112:6789-95.

Key AP, Jones D, Dykens EM (2013) Social and emotional processing in Prader-Willi syndrome:genetic subtype differences $J$ of Neurodevelopmental Disorders 5:7-12

Koenig K, Klin A, Schultz R (2004) Deficits in social attribution theory in Prader-Willi syndrome J Autism Dev Dis 34:573-582

Leekam, S., Baron-Cohen, S., Brown, S., Perrett, D., \& Milders, M. (1997). Eye-direction detection:A dissociation between geometric and joint-attention skills in autism. British Journal of Developmental Psychology, 15: 77-95.

Lo ST, Siemensma E, Collin P, Hokken-Koelega A (2013) Impaired theory of mind and symptoms of Autism Spectrum Disorder in children with Prader-Willi syndrome. Res in Dev Disabil.34:2764-73

Lucignani G, Panzacchi A, Bosio L, Moresco RM, Ravasi L, Coppa I, Chiumello G, Frey K, Koeppe R, Fazio F (2004) $\mathrm{GABA}_{\mathrm{A}}$ receptor abnormalities in Prader-Willi syndrome assessed with positron emission tomography and $\left[{ }^{11} \mathrm{C}\right]$ flumazenil NeuroImage 22:22-28

Lukoshe A, Hokken-Koelega AC, van der Lugt A, White T (2014) Reduced cortical complexity in children with Prader-Willi syndrome and its association with cognitive impairment and developmental delay. PLoS ONE 9:e107320

Malich S, Largo RH, Schinzel A, Molinari L, Eiholzer U (2000) Phenotypic heterogeneity of growth and psychometric intelligence in Prader-Willi syndrome:variable expression of a contiguous gene syndrome or parent-child resemblance? Am J Med Genet 91:298-304

Manning, K., E., \& Holland, A., J. (2015). Puzzle Pieces: Neural Structure and Function in Prader-Willi Syndrome. Diseases, 3(4), 382-415.

Meziane H, Schaller F, Bauer S, Villard C, Materazzo V, Riet F, Guillon G, Lafitte D, Desarmenien MG, Tauber M, Muscatelli F (2015) An early postnatal oxytocin treatment prevents social and learning deficits in adult mice deficient for Magel2, a gene involved in Prader-Willi syndrome and autism Biolog Psychiat 78:85-94 
Miller JL, Kranzler J, Liu Y, Schmalfuss I, Theriaque DW, Shuster JJ, Hatfield A, Mueller OT, Goldstone AP, Sahoo T, Beaudet AL, Driscoll DJ (2006) Neurocognitive findings in Prader-Willi syndrome and early onset morbid obesity J Pediatr. 149:192-8.

Miller JL, Couch JA, Schmalfuss H, He G, Liu Y, Driscoll DJ (2007) Intracranial abnormalities detected by three-dimensional magnetic resonance imaging in Prader-Willi syndrome Am J Med Genet A 143A:476-483

Muscatelli F, Abrous DN, Massacrier A, Boccaccio I, Le Moal M, Cau P, Cremer H (2000) Disruption of the mouse Necdin gene results in hypothalamic and behavioral alterations reminiscent of the human Prader-Willi syndrome. Hum Mol Genet 9:3101-10

Ogata H, Ihara H, Murakami N, Gito M, Kido Y, Nagai T (2014) Autism spectrum disorders and hyperactive/impulsive behaviors in Japanese patients with Prader-Willi syndrome: a comparison between maternal uniparental disomy and deletion cases. Am J Med Genet Part A 164A:2180-86

Pangelinan MM, Zhang G,Vanmeter JW, Clark JE, Hatfield BD, Haufler AJ, (2011) Beyond age and gender: relationships between cortical and subcortical brain volume and cognitive-motor abilities in school-age children NeuroImage 54:3093-3100

Paul SM (1980) Sibling resemblance in mental ability: a review. Behavior Genetics 10:277-289

Relkovic D, Doe CM, Humby T, Johnstone KA, Resnic JL, Holland AJ, Hagan JJ, Wilkinson LS, Isles AR (2010) Behavioural and cognitive abnormalities in an imprinting centre deletion mouse model for Prader-Willi syndrome Euro J Neurosci 31:156-164

Relkovic D, Humby T, Hagan JJ. Wilkinson LS, Isles AR (2012) Enhanced appetitive learning and reversal learning in a mouse model for Prader-Willi syndrome. Behav Neurosci 126:488-92

Semenza C, Pignatti R, Bertella L, Ceriani F, Mori I, Molinari E, Giardino D, Malvestiti F, Grugni G (2008) Genetics and mathematics: evidence from Prader-Willi syndrome. Neuropsychologia 46:206-12

Stauder JE, Boer H, Gerits RH, Tummers A, Whittington J, Curfs LM. (2005) Differences in behavioural phenotype between parental deletion and maternal uniparental disomy in Prader-Willi syndrome: an ERP study. Clinical Neurophysiology 116:1464-70.

Steerneman, P.M. C.. (2009). ToM test-R handleiding. Antwerpen-Apeldoorn: Garant.

Sullivan K, Tager-Flusberg H (1999) Second-order belief attribution in Williams syndrome: intact or impaired? Am J Ment Retard 104:523-32

Tager-Flusberg H, Sullivan K (2000) A componential view of theory of mind: evidence from Williams syndrome Cognition 76:59-89

Verdine BN, Troseth GL, Hodapp RM, Dykens EM. (2008) Strategies and correlates of jigsaw puzzle and visuospatial performance by persons with Prader-Willi syndrome. Am. J. Ment. Retard. 113:343-55.

Walley RM, Donaldson MD (2005) An investigation of executive function abilities in adults with Prader-Willi syndrome. Journal of Intellectual Disability Research 49:613-625.

Whittington JE, Holland AJ, Webb T, Butler JV, Clarke DJ, Boer H. (2004a). Cognitive abilities and genotype in a population-based sample of people with Prader-Willi syndrome. Journal of Intellectual Disability Research, 48:172-187. 
Whittington JE, Holland AJ, Webb T, Butler JV, Clarke DJ, Boer H. (2004b)

Underachievement in Prader-Willi Syndrome. Journal of Intellectual Disability Research 48:188-200

Whittington JE and Holland AJ. (2011) Recognition of emotion in facial expression by people with Prader-Willi syndrome. Journal of Intellectual Disability Research 55:75-84.

Whittington JE, Holland AJ, Webb T. (2009) Relationship between the IQ of people with Prader-Willi syndrome and that of their siblings: evidence for imprinted gene effects. Journal of Intellectual Disability Research. 53:411-418.

Whittington JE, Holland AJ, Webb T, Butler JV, Clarke DJ, Boer H. (2002) Relationship between clinical and genetic diagnosis of Prader-Willi syndrome. Journal of Medical Genetics 39:926-932.

Woodcock K, Oliver C, Humphreys G. (2009a) Associations between repetitive questioning, resistance to change, temper outbursts and anxiety in Prader-Willi and Fragile-X syndromes. J. Intellect. Disabil. Res. 53:265-78.

Woodcock KA, Oliver C, Humphreys GW. (2009b) Task-switching deficits and repetitive behaviour in genetic neurodevelopmental disorders: data from children with PraderWilli syndrome chromosome 15 q11-q13 deletion and boys with Fragile X syndrome. Cogn. Neuropsychol. 26:172-94.

Woodcock KA, Oliver C, Humphreys GW. (2011) The relationship between specific cognitive impairment and behaviour in Prader-Willi syndrome. J. Intellect. Disabil. Res. 55:152-71.

Yamada K, Matsuzawa H, Uchiyama M, Kwee IL, Nakada T (2006) Brain developmental abnormalities in Prader-Willi syndrome detected by diffusion tensor imaging Pediatrics 118:e442-8 
Figure 1

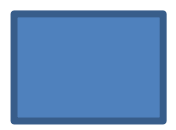

Maternally imprinted (paternally expressed)

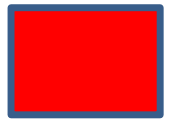

Paternally imprinted (maternally expressed) 